\title{
Triptolide suppresses ultraviolet B-enhanced sebum production by inhibiting the biosynthesis of triacylglycerol in hamster sebaceous glands in vivo and in vitro
}

\author{
TAKASHI SATO ${ }^{1}$, NORIKO AKIMOTO ${ }^{1}$, AIKO TAKAHASHI $^{1}$ and AKIRA ITO ${ }^{2}$ \\ ${ }^{1}$ Department of Biochemistry, School of Pharmacy; ${ }^{2}$ The Institute for Social Medicine, \\ Tokyo University of Pharmacy and Life Sciences, Hachioji, Tokyo 192-0392, Japan
}

Received October 26, 2015; Accepted February 24, 2017

DOI: $10.3892 /$ etm.2017.4461

\begin{abstract}
Ultraviolet B (UVB) irradiation causes alterations in cutaneous barrier function, including excessive production of sebum in sebaceous glands, which is associated with the aggravation of acne. This study aimed to evaluate the inhibitory effects of triptolide, a diterpenoid triepoxide from Tripterygium wilfordii Hook F, on sebocytic lipogenesis in UVB-irradiated hamster skin in vivo and in vitro. Topical application of triptolide decreased the UVB-enhanced sebum accumulation in the sebaceous glands of hamster skin. The level of triacylglycerol (TG), a major sebum component, on the skin surface was reduced by triptolide treatment in UVB-irradiated hamsters, whereas there was no change in that of free-fatty acids and cholesterol, which are minor sebum components. UVB irradiation significantly enhanced TG production $(\mathrm{P}<0.01$ in extracellular lipids, $\mathrm{P}<0.05$ in intracellular lipids), and the activity of acyl coenzyme A/diacylglycerol acyltransferase (DGAT), a rate-limiting enzyme of TG synthesis, in differentiated hamster sebocytes $(\mathrm{P}<0.05$ at $6 \mathrm{~h}$ and UVB of $0.62 \mathrm{~kJ} / \mathrm{m}^{2}, \mathrm{P}<0.001$ at $24 \mathrm{~h}$ and UVB 0.37 or $\left.0.62 \mathrm{~kJ} / \mathrm{m}^{2}\right)$. Furthermore, triptolide significantly inhibited UVB-enhanced TG production $(\mathrm{P}<0.05$ at $28 \mathrm{nM}$ and $\mathrm{P}<0.01$ at 56 and $112 \mathrm{nM}$ triptolide) and DGAT activity $(\mathrm{P}<0.01$ at $28 \mathrm{nM}$ and $\mathrm{P}<0.001$ at 56 and $112 \mathrm{nM}$ triptolide) in differentiated hamster sebocytes. These results provide novel evidence that triptolide decreases UVB-enhanced sebum production by inhibiting DGAT-dependent TG biosynthesis in differentiated hamster sebocytes. These findings may be applicable to the prevention of acne aggravation.
\end{abstract}

Correspondence to: Professor Takashi Sato, Department of Biochemistry, School of Pharmacy, Tokyo University of Pharmacy and Life Sciences, 1432-1 Horinouchi, Hachioji, Tokyo 192-0392, Japan

E-mail: satotak@toyaku.ac.jp

Key words: triptolide, ultraviolet B irradiation, sebaceous glands, hamster sebocytes, triacylglycerol biosynthesis, acyl coenzyme A/diacylglycerol acyltransferase, Tripterygium wilfordii Hook F

\section{Introduction}

Ultraviolet B (UVB) irradiation induces acute and chronic skin disorders, characterized by inflammation such as erythema and edema, hyperplasia, dyspigmentation and deep wrinkle formation (termed photoaging) (1-3). These clinical symptoms have been reported to contribute to the functional and structural abnormalities of the epidermis and dermis, including an increase of epidermal thickness, a decrease of epidermal polarity and the augmented degradation of extracellular matrices such as collagen and elastin (4-6). On the other hand, sebum secretion is considered to be involved in the functional maintenance of the cutaneous surface as a biologic barrier (7). Excessive sebum production and secretion in sebaceous glands has been reported to be associated with the development and aggravation of acne vulgaris and seborrheic dermatitis (8). In addition, UVB-irradiation has been reported to increase sebum secretion and production in humans and hamsters in vivo (9-11). Furthermore, UVB exposure has been reported to facilitate free radical-mediated lipid peroxidation of sebum in the skin $(10,12)$. Therefore, sunscreen or topical and systemic administration of antioxidants is likely to be an efficient strategy for preventing the UV-mediated perturbation of cutaneous barrier functions (13).

Extracts of the Chinese herb, Tripterygium wilfordii Hook $\mathrm{F}$ (TWHF), have been reported to be effective in the treatment of autoimmune diseases such as rheumatoid arthritis, nephritis and lupus erythematosus (14-16). Triptolide, a diterpenoid triepoxide, has been reported to be a component of TWHF that possesses immunosuppressive, anti-inflammatory and anti-oxidative actions in vivo and in vitro (17-20). A previous study by the current authors reported that triptolide inhibits the production of prostaglandin E2 due to the transcriptional suppression of prostaglandin $\mathrm{H}$ synthase (cyclooxygenase) 2 in human synovial fibroblasts and mouse macrophages (18). In addition, prostaglandin $\mathrm{E} 2$ has been reported to be a prominent indicator of UVB-mediated inflammation in the skin, which contributes to the regulation of keratinocyte proliferation and differentiation (21). Therefore, triptolide may be a candidate for a novel therapeutic agent that improves skin barrier functions under UVB-mediated acute and chronic inflammatory conditions, including acne. However, it is not yet fully understood 
whether triptolide can control the UVB-mediated dysregulation of sebum production in sebaceous glands.

In the present study, the effects of triptolide on UVB-augmented sebum production in sebaceous glands in hamsters were examined both in vivo and in vitro. The results indicated that triptolide inhibits UVB-enhanced sebum production in sebaceous glands.

\section{Materials and methods}

Preparation of differentiated hamster sebocytes. Hamster sebocytes were established from the sebaceous glands of auricles of five-week-old male golden hamsters (Japan SLC, Inc., Hamamatsu, Japan), as described previously (22). Hamster sebocytes $\left(2.4 \times 10^{4}\right.$ cells $\left./ \mathrm{cm}^{2}\right)$ were plated onto 12 -well plates or 35-mm diameter culture dishes (BDBiosciences, Franklin Lakes, NJ, USA), then cultured for $24 \mathrm{~h}$ at $37^{\circ} \mathrm{C}$ in Dulbecco's modified Eagle's medium/F12 (Invitrogen; Thermo Fisher Scientific, Inc., Waltham, MA, USA) supplemented with $6 \%$ heat-denatured fetal bovine serum (Sigma-Aldrich; Merck KGaA, Darmstadt, Germany), 2\% human serum (ICN Biomedicals, Irvine, CA, USA), $0.68 \mathrm{mM} L$-glutamine (Invitrogen; Thermo Fisher Scientific, Inc.) and $10 \mathrm{nM}$ recombinant human epidermal growth factor (Progen Biotechnik GmbH, Heidelberg, Germany) to achieve complete cell adhesion, as previously described (11). The hamster sebocytes were treated every two days for up to 10 days with $10 \mathrm{nM}$ insulin (Sigma-Aldrich; Merck KGaA), during which time intracellular lipid droplets were abundantly formed. The resulting cells were termed differentiated hamster sebocytes (DHS) (11). In this series of experiments, hamster sebocytes were used up to the third passage level.

In vivo and in vitro UVB irradiation and triptolide treatment. The auricle skin of three-week-old male golden hamsters $(n=6$; weight, 48.7 $\pm 3.3 \mathrm{~g}$; Japan SLC, Inc.) was topically treated once a day with $50 \mu \mathrm{l}$ of a $3.1 \mathrm{nmol}$ solution of triptolide (Enzo Life Sciences, Inc., Farmingdale, NY, USA; n=3; Fig. 1) in 95\% ethanol and 5\% glycerol, or with the same volume of vehicle $(n=3)$ before each UVB irradiation $\left(5.4 \mathrm{~kJ} / \mathrm{m}^{2}\right)$, once a day for seven days (11). For the in vitro UVB irradiation experiments, DHS in 12-well plates were irradiated with UVB $\left(0.12-0.62 \mathrm{~kJ} / \mathrm{m}^{2}\right)(6)$ in a serum-free culture medium supplemented with triptolide $(28-112 \mathrm{nM})$, then cultured for $24 \mathrm{~h}$ at $37^{\circ} \mathrm{C}$. UVB irradiation was performed using a Toshiba FL20S fluorescent sunlamp (Toshiba Corporation, Tokyo, Japan), emitting rays between 275 and $375 \mathrm{~nm}$ with a peak emission of $313 \mathrm{~nm}$. The radiance was measured by a UV Indicator MI-340 (EKO Instruments, Tokyo, Japan), as previously described (6). Hamsters were housed in a room maintained at $23 \pm 1^{\circ} \mathrm{C}$ and $55 \pm 5 \%$ humidity with a 12-h light/dark cycle (lights on 7:00 a.m. to 7:00 p.m.) during the experiments. Food and water were available ad libitum. All experiments presented in this study were performed according to the Guidelines of Experimental Animal Care issued by the Prime Minister's Office of Japan (Tokyo, Japan). The experimental protocol was approved by the Committee of Animal Care and Use of Tokyo University of Pharmacy and Life Sciences (Tokyo, Japan).

Oil red $\mathrm{O}$ staining. Animals were sacrificed by $\mathrm{CO}_{2}$ inhalation. Auricle skin from hamsters irradiated with or without

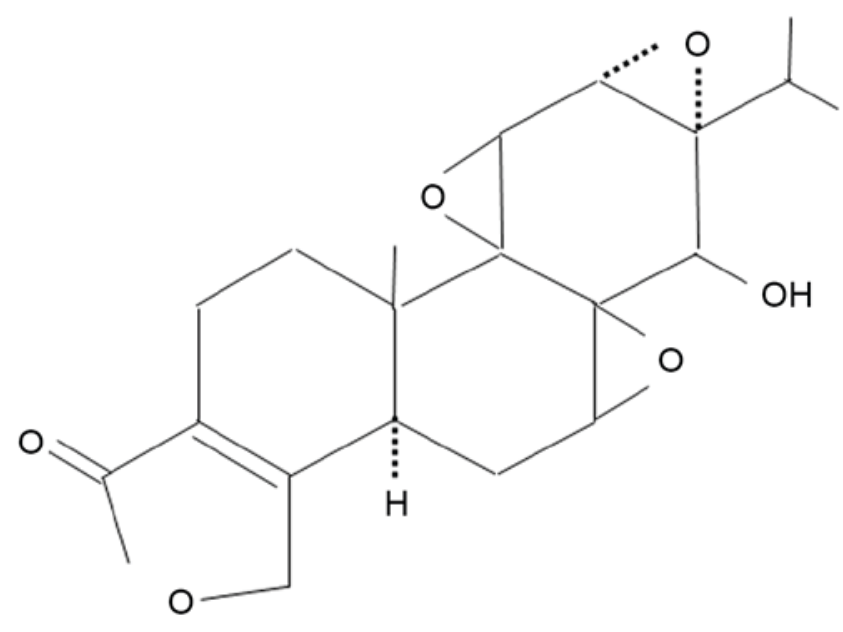

Figure 1. Chemical structure of triptolide.

UVB as described above was snap-frozen in liquid nitrogen. The frozen tissue sections $(8-\mu \mathrm{m})$ were incubated in $60 \%$ isopropanol after washing with distilled $\mathrm{H}_{2} \mathrm{O}$. Tissue sections were stained with $0.3 \%$ oil red O (Sigma-Aldrich; Merck $\mathrm{KGaA})$ in a solution of isopropanol and distilled $\mathrm{H}_{2} \mathrm{O}(3: 2$, vol:vol) at $37^{\circ} \mathrm{C}$ for $15 \mathrm{~min}$. Sections were then viewed with a light microscope furnished with a digital camera (Olympus Corporation, Tokyo, Japan). Sections were also counterstained with Mayer's hematoxylin solution (Wako Pure Chemical Industries, Ltd., Osaka, Japan), as previously described (23).

Analysis of sebum components. Lipid components of sebum from the skin surface of hamster auricles and in cultured hamster sebocytes were analyzed using an automatic thin-layer chromatography (TLC) using a flame ionization detector (Iatroscan; Iatron Laboratories, Inc., Tokyo, Japan), as previously described (22). Briefly, the auricles were wiped with acetone-impregnated cotton, then the sebum on the skin surface was extracted twice for $30 \mathrm{sec}$ with $50 \mu 1$ of acetone in stainless steel cups, $1 \mathrm{~h}$ after the wiping. The cells were harvested with $0.25 \%$ trypsin and $0.02 \%$ EDTA in phosphate-buffered saline, then sonicated. The cell lysate and harvested culture medium were mixed with chloroform:methanol (2:1) for $5 \mathrm{~min}$ at room temperature. The mixture was separated by centrifugation at $1,000 \mathrm{x} \mathrm{g}$ for $5 \mathrm{~min}$ at room temperature following addition of $0.88 \%$ $\mathrm{KCl}$. After removing the methanol (the upper phase) with a pipette, the chloroform fraction (the lower phase), including lipids, was collected. The sebum extracts were subjected to Iatroscan and the levels of TG, a major sebum component, and minor sebum components, free-fatty acids (FFAs) and cholesterol $(\mathrm{Ch})$, were calculated using an internal control, authentic cholesterol acetate $(2 \mathrm{~g}$; Doosan Serdary Research Laboratories, Toronto, Canada). Tripalmitin (for TG), palmitic acid (for FFA) and Ch (Doosan Serdary Research Laboratories) were used as lipid standards for chromatography.

Measurement of intracellular TG. After the treatment of DHS with UVB and/or triptolide, the harvested cells 
A

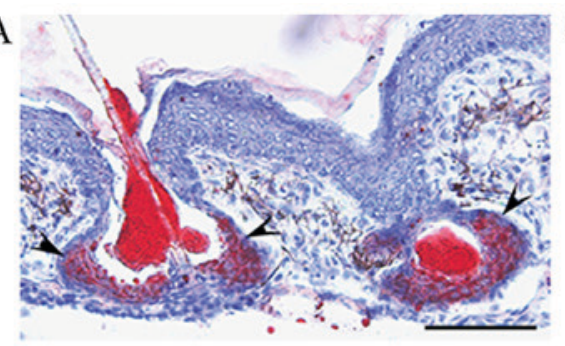

B

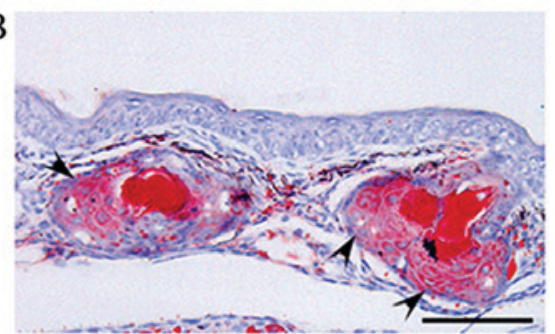

$\mathrm{C}$

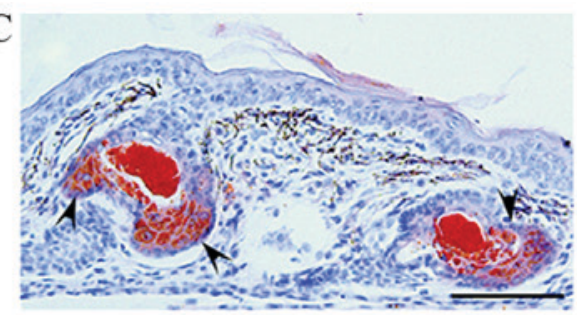

Figure 2. Effects of UVB-irradiation and external triptolide application on sebaceous glands in hamsters. Auricles of hamsters were treated once a day with triptolide $(3.1 \mathrm{nmol}) / 95 \%$ ethanol/5\% glycerol $(\mathrm{n}=3)$ or vehicle $(95 \%$ ethanol $/ 5 \%$ glycerol; $\mathrm{n}=3)$ prior to UVB irradiation at $5.4 \mathrm{~kJ} / \mathrm{m}^{2}$. After seven days, the tissues were fixed and stained with oil red O. (A) Vehicle treatment. (B) UVB irradiation. (C) Triptolide treatment and UVB irradiation. Arrowheads indicate sebaceous glands. Bars, $100 \mu \mathrm{m}$. UVB, ultraviolet B.

were subjected to quantification of TG using Liquitech TG-II (Roche Diagnostics K.K., Tokyo, Japan), as previously described (11). The quantity of intracellular TG was calculated using an authentic trioleinate-standard solution (0.6 mg/ml).

Acyl coenzyme A (coA)/diacylglycerol acyltransferase (DGAT) activity. Acyl CoA/DGAT activity in DHS treated with UVB and/or triptolide was measured using 1,2-dioleoyl glycerol (Cayman Chemical Co., Ann Arbor, MI, USA) and ${ }^{14} \mathrm{C}$-palmitoyl-CoA (GE Healthcare Biosciences, Pittsburgh, PA, USA), as previously described (24).

Statistical analysis. Data are presented as the mean \pm standard deviation, and were analyzed using one-way analysis of variance, and the Fisher test for multiple comparisons. SPSS 17.0 software was used to analyze the data (SPSS, Inc., Chicago, IL, USA). $\mathrm{P}<0.05$ was considered to indicate a statistically significant difference.

\section{Results}

Effects of topical application of triptolide on sebum levels on the skin surface. The effects of triptolide on sebum accumulation were evaluated in sebaceous glands in three-week-old male golden hamsters. Oil red $\mathrm{O}$ staining revealed that sebum accumulation in the sebaceous glands and follicular ducts, as well as sebaceous gland size, were increased in the UVB-irradiated hamsters compared with the control (Fig. 2A and $\mathrm{B})$. In addition, when triptolide (3.1 nmol) was topically applied to the skin of auricles before each UVB-irradiation, the UVB-enhanced sebum accumulation and sebaceous size were decreased (Fig. 2B and C). Therefore, these results suggested that topical application of triptolide reduced UVB-induced aberrant sebum accumulation in sebaceous glands in vivo.

Characterization of skin surface lipid components in triptolide-treated hamsters. It was previously reported by the current authors that sebum produced in hamster sebocytes consists of TG, the major lipid component, and other minor components such as FFAs and Ch (22). Therefore, in the current study, alterations of sebum components on the skin surface were evaluated in UVB and/or triptolide-treated hamsters. A TLC analysis of sebum on the skin surface
Table I. Lipid composition on the skin surface of auricles in UVB-irradiated hamsters.

Relative amounts of lipids (fold vs. control)

\begin{tabular}{lccc}
\cline { 2 - 4 } Treatment & TG & FFAs & Ch \\
\hline Control & 1.0 & 1.0 & 1.0 \\
UVB $\left(5.4 \mathrm{~kJ} / \mathrm{m}^{2}\right)$ & $2.3 \pm 1.2^{\mathrm{a}}$ & $1.5 \pm 0.3^{\mathrm{a}}$ & $2.0 \pm 0.5^{\mathrm{a}}$ \\
\hline
\end{tabular}

Lipids on the skin surface of auricles in Fig. 2 were extracted and subjected to thin layer chromatographic analysis. Data are presented as the mean \pm standard deviation from three individual extracts. ${ }^{\mathrm{a}} \mathrm{P}<0.05$ vs. untreated hamsters (control). UVB, ultraviolet $\mathrm{B} ; \mathrm{TG}$, triacylglycerol; FFAs, free fatty acids; Ch, cholesterol.

indicated that UVB irradiation significantly augmented the levels of TG $(2.3 \pm 1.2$-fold, $\mathrm{P}<0.05)$, FFAs $(1.5 \pm 0.3$-fold, $\mathrm{P}<0.05)$ and $\mathrm{Ch}(2.0 \pm 0.5$-fold, $\mathrm{P}<0.05)$ (Table I). In addition, topical application of triptolide was found to significantly decrease the level of TG $(83 \%$ inhibition, $\mathrm{P}<0.05)$ compared with control hamsters treated with a vehicle (Fig. 3). However, there was little change in the level of FFAs and $\mathrm{Ch}$ on the skin surface of UVB-irradiated hamsters compared with controls. These results suggested that triptolide selectively modulated the level of TG rather than other sebum components in the skin of UVB-irradiated hamsters.

Effect of UVB irradiation on sebaceous lipogenesis and $D G A T$ activity in DHS. In order to clarify the molecular mechanisms by which triptolide selectively decreased the TG level on the skin surface of UVB-irradiated hamsters, the regulation of TG production and DGAT expression was evaluated in UVB-irradiated DHS. When the DHS were irradiated with UVB $\left(0.62 \mathrm{~kJ} / \mathrm{m}^{2}\right)$ and cultured for $24 \mathrm{~h}$, TLC analysis indicated that the extracellular levels of TG, FFAs and $\mathrm{Ch}$ were significantly increased compared with non-irradiated controls (Fig. 4A). In addition, UVB irradiation of DHS was indicated to significantly increase the intracellular levels of TG and FFAs compared with non-irradiated controls, but had no significant effect on the levels of Ch (Fig. 4B). This indicated that UVB irradiation increased the levels of TG in DHS. Furthermore, the enzymatic activity of DGAT, a rate-limiting 


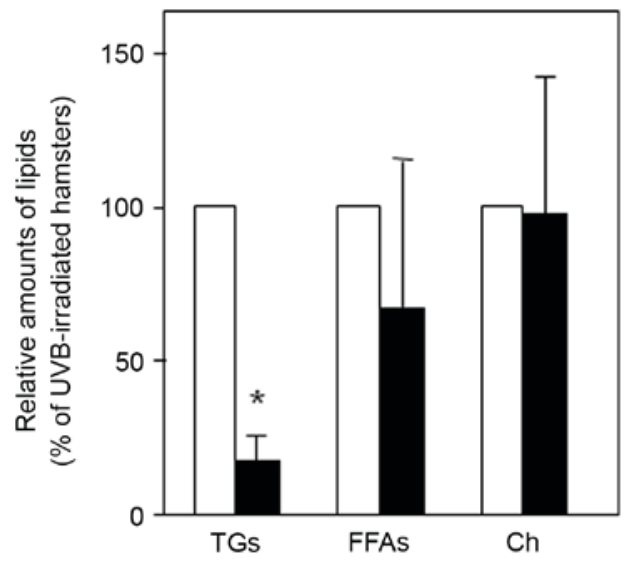

Figure 3. Effect of triptolide on the levels of skin surface lipids in auricles from UVB-irradiated hamsters. The lipids on the skin surface of the vehicle- (white columns) and triptolide-treated (black columns) auricles of UVB-irradiated hamsters were extracted and subjected to TLC analysis. Data are presented as the mean \pm standard deviation from three individual extracts. ${ }^{\prime} \mathrm{P}<0.05$ vs. vehicle-treated hamsters. TG, triacylglycerol; FFAs, free fatty acids; Ch, cholesterol; UVB, ultraviolet B; TLC, thin-layer chromatography; TG, triacylglycerol; FFAs, free fatty acids; $\mathrm{Ch}$, cholesterol.
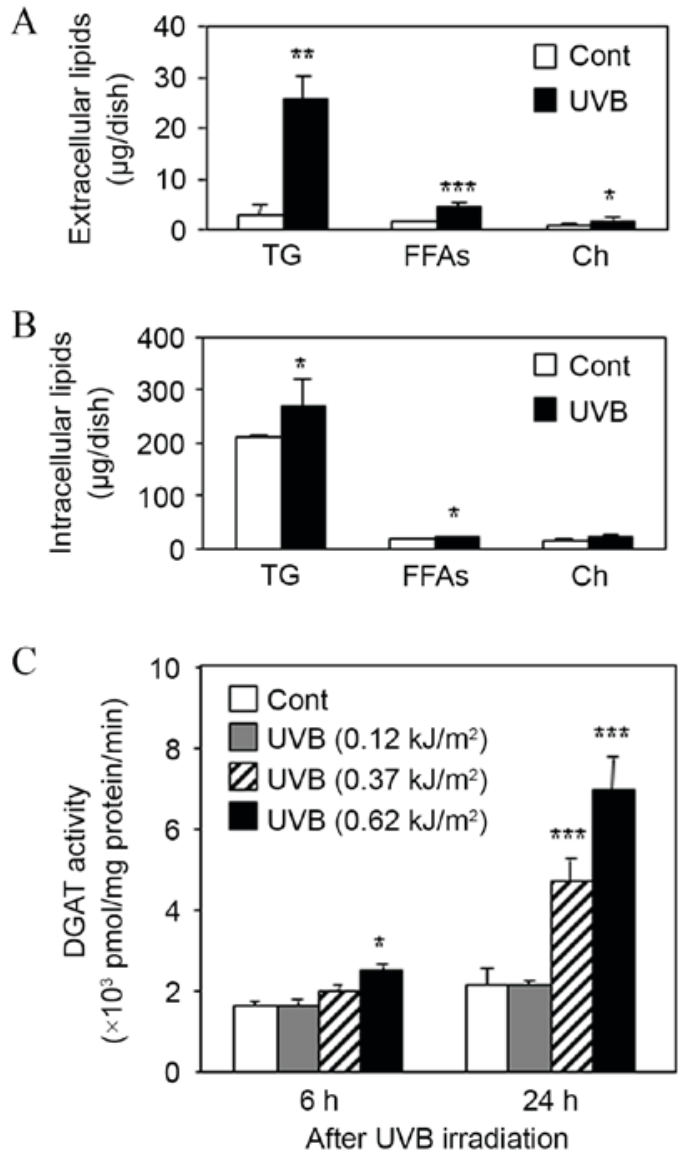

Figure 4. Effect of UVB irradiation on sebaceous lipogenesis and DGAT activity in DHS. DHS at the third passage were irradiated with UVB $\left(0.62 \mathrm{~kJ} / \mathrm{m}^{2}\right)$ in serum-free conditions and then maintained for $24 \mathrm{~h}$. (A) Harvested culture media and (B) cells were subjected to TLC analysis. (C) DHS at the third passage were irradiated with UVB at 0.12, 0.37 and $0.62 \mathrm{~kJ} / \mathrm{m}^{2}$. After the irradiation, the cells were maintained for 6 or $24 \mathrm{~h}$, then DGAT activity was measured in the harvested cells. Data are presented as the mean \pm standard deviation of three dishes. ${ }^{*} \mathrm{P}<0.05,{ }^{* *} \mathrm{P}<0.01$ and ${ }^{* * *} \mathrm{P}<0.01$ vs. untreated controls. DHS, differentiated hamster sebocytes; TLC, thin-layer chromatography; DGAT, diglyceride acyltransferase; UVB, ultraviolet B; TG, triacylglycerol; FFAs, free fatty acids; $\mathrm{Ch}$, cholesterol.
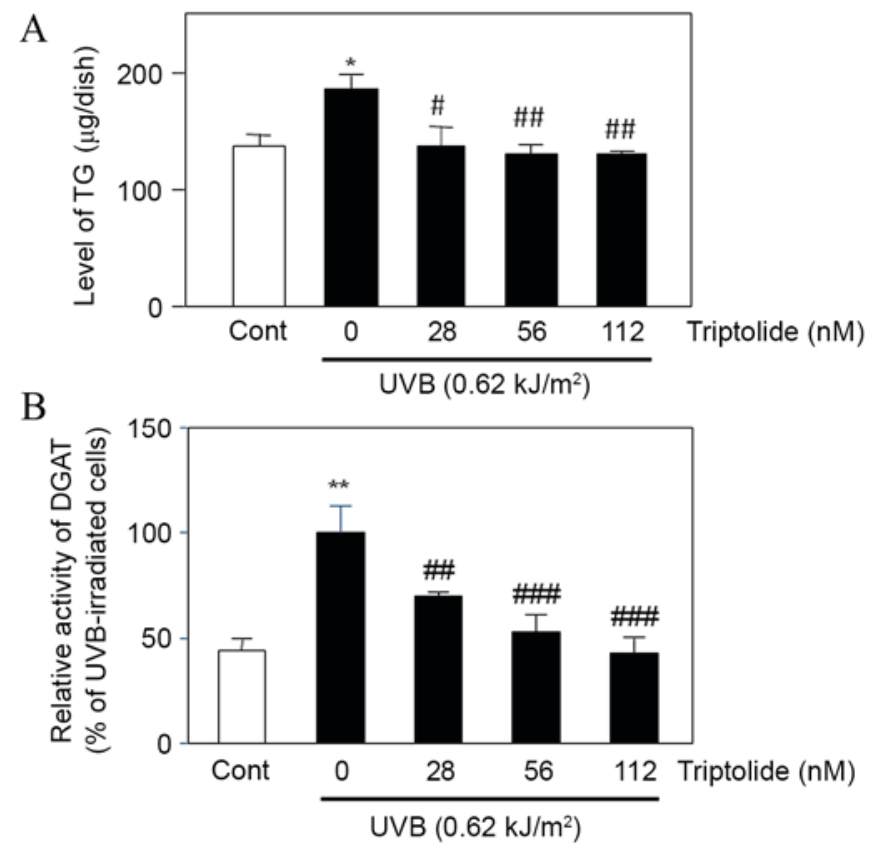

Figure 5. Effect of triptolide on UVB-enhanced TG production and DGAT activity in DHS. DHS at the third passage were treated with triptolide $(28-112 \mathrm{nM})$ prior to UVB irradiation $\left(0.62 \mathrm{~kJ} / \mathrm{m}^{2}\right)$, then maintained for $24 \mathrm{~h}$. (A) TG and (B) DGAT activity was measured in the harvested cells Data are presented as the mean \pm standard deviation of three dishes. ${ }^{*} \mathrm{P}<0.05$ and ${ }^{* *} \mathrm{P}<0.01$ vs. untreated controls; ${ }^{\#} \mathrm{P}<0.05,{ }^{\# \#} \mathrm{P}<0.01$ and ${ }^{\# \# \#} \mathrm{P}<0.001$ vs UVB-irradiated cells. DHS, differentiated hamster sebocytes; TG, triacylglycerol; DGAT, diglyceride acyltransferase; UVB, ultraviolet B.

enzyme of TG synthesis, was dose-dependently increased by UVB irradiation in DHS (Fig. 4C).

Effect of triptolide on UVB-enhanced TG production and DGAT activity in DHS. Treatment of UVB-irradiated DHS with triptolide was found to significantly decrease the intracellular level of TG $(\mathrm{P}<0.05$ at $28 \mathrm{nM}, \mathrm{P}<0.01$ at 56 and $112 \mathrm{nM}$ triptolide; Fig. 5A) compared with the non-treated cells. Triptolide also significantly decreased DGAT activity in UVB-irradiated triptolide-treated cells compared with UVB-irradiated non-treated cells $(\mathrm{P}<0.01$ at $28 \mathrm{nM}, \mathrm{P}<0.001$ at 56 and $112 \mathrm{nM}$ triptolide; Fig. 5B). These results suggested that triptolide suppressed the UVB-enhanced DGAT activity and TG production in DHS.

\section{Discussion}

UVB-irradiation has been reported to facilitate sebum secretion and production in vivo $(9,10)$, yet it remains unclear how UVB stimulates sebum production in sebaceous glands. To the best of our knowledge, the present study is the first to demonstrate that UVB irradiation increases DGAT activity, leading to an increase in TG production in DHS. Taken together with a previous report using DGAT knockout mice, in which DGAT was shown to be involved in TG biosynthesis and lipid-droplet formation in adipocytes (25). UVB is likely to facilitate sebum production due to DGAT-mediated de novo synthesis of TG in DHS. Furthermore, as most people are exposed to UV in sunlight on an everyday basis (3), it is not only endogenous factors, such as androgens and insulin, that serve key roles in the 
regulation of sebum production and secretion, but potentially also environmental ones. Moreover, excess and/or long-term UVB irradiation is likely to perturb epidermal barrier functions through the abnormal enhancement of sebum production and secretion, as well as alterations in the expression pattern of tight junction-related molecules (26).

Akitomo et al (10) reported that UV exposure facilitates the peroxidation of skin surface lipids such as TG and $\mathrm{Ch}$, which in turn affects the cutaneous barrier functions by increasing transepidermal water loss. Regarding the anti-oxidative actions of triptolide, previous studies $(19,20)$ have reported that triptolide exhibits anti-oxidative actions due to the inhibition of reactive oxygen species levels in the mouse liver, and superoxide anions in murine peritoneal macrophages. In addition, the current authors previously demonstrated that a natural anti-oxidative polymethoxy flavonoid, nobiletin, predominantly decreases the level of $\mathrm{TG}$, which is the target lipid for UVB-peroxidation (11). Therefore, it is suggested that the topical application of triptolide is effective for the prevention of sebum peroxidation on the skin, not only due to its own anti-oxidative activity but also due its ability to inhibit sebaceous TG synthesis.

In the present study, it was indicated that the extracellular level of TG was increased in UVB-irradiated DHS, suggesting that sebum excretion in DHS is facilitated by UVB. Since sebum secretion is generally considered to be regulated by a holocrine mechanism, which may involve the apoptosis of sebocytes (27), it was confirmed that there were no apoptotic cells in this experimental condition (data not shown). In addition, the current authors have previously reported on the apoptosis-independent and ATP-binding cassette (ABC) transporter (ABCB1/P-glycoprotein) -mediated TG secretion in DHS (28). Regarding the relationship between ABCB1 activity and UV irradiation, Trindade et al (29) have reported that ABCB1-overexpressing leukemia cells with a multidrug-resistance phenotype are resistant to UVA but sensitive to UVB and UVC. Further experiments are required to clarify whether ABCB1 is associated with the UVB-increased TG excretion in DHS.

Acne vulgaris is characterized by: i) Excess sebum production in sebaceous glands; ii) microcomedone formation; and iii) the hyperproliferation of Propionibacterium acnes, which results in inflammatory conditions in acne lesions (8). The aggravation of acne is likely to result in a disfiguring and permanent scar formation that carries a psychological and social impact on the patient's quality of life $(8,30,31)$. Allen and LoPresti (32) reported the involvement of sunlight in the aggravation of acne vulgaris, and the current results indicated that triptolide inhibits UVB-enhanced sebaceous TG production in vivo and in vitro. These findings suggest that triptolide could be used in the future as a therapeutic agent or in cosmetics, for the effective prevention of sunlight-associated acne aggravation.

In conclusion, the present study demonstrated that triptolide inhibits UVB-induced sebaceous lipogenesis in hamsters both in vivo and in vitro. Furthermore, the study has supplied novel evidence indicating that triptolide decreases UVB-augmented TG production by suppressing DGAT activity in DHS. Thus, triptolide is a candidate to be used as a therapeutic agent or in cosmetics for acne treatment.

\section{Acknowledgements}

This study was supported by a Grant-in-Aid for Scientific Research (C) (grant no. 26460633) awarded to Professor Takashi Sato.

\section{References}

1. Rabe JH, Mamelak AJ, McElgunn PJ, Morison WL and Sauder DN: Photoaging: Mechanisms and repair. J Am Acad Dermatol 55: 1-19, 2006.

2. Quan T, Qin Z, Xia W, Shao Y, Voorhees JJ and Fisher GJ: Matrix-degrading metalloproteinases in photoaging. J Invest Dermatol Symp Proc 14: 20-24, 2009.

3. Sambandan DR and Ratner D: Sunscreens: An overview and update. J Am Acad Dermatol 64: 748-758, 2011.

4. Jenkins G: Molecular mechanisms of skin aging. Mech Ageing Dev 123: 801-810, 2002

5. Inomata S, Matsunaga Y, Amano S, Takada K, Kobayashi K, Tsunenaga M, Nishiyama T, Kohno Y and Fukuda M: Possible involvement of gelatinases in basement membrane damage and wrinkle formation in chronically ultraviolet $\mathrm{B}$-exposed hairless mouse. J Invest Dermatol 120: 128-134, 2003.

6. Tanaka S, Sato T, Akimoto N, Yano M and Ito A: Prevention of UVB-induced photoinflammation and photoaging by a polymethoxy flavonoid, nobiletin, in human keratinocytes in vivo and in vitro. Biochem Pharmacol 68: 433-439, 2004.

7. Thody AJ and Shuster S: Control and function of sebaceous glands. Physiol Rev 69: 383-416, 1989.

8. Kurokawa I, Danby FW, Ju Q, Wang X, Xiang LF, Xia L, Chen W, Nagy I, Picardo M, Suh DH, et al: New developments in our understanding of acne pathogenesis and treatment. Exp Dermatol 18: 821-832, 2009.

9. Suh DH, Kwon TE and Youn IJ: Changes of comedonal cytokines and sebum secretion after UV irradiation in acne patients. Eur J Dermatol 12: 139-144, 2002.

10. Akitomo Y, Akamatsu H, Okano Y, Masaki H and Horio T: Effects of UV irradiation on the sebaceous gland and sebum secretion in hamsters. J Dermatol Sci 31: 151-159, 2003.

11. Sato T, Takahashi A, Kojima M, Akimoto N, Yano M and Ito A: A citrus polymethoxy flavonoid, nobiletin inhibits sebum production and sebocyte proliferation, and augments sebum excretion in hamsters. J Invest Dermatol 127: 2740-2748, 2007.

12. Merle C, Laugel C and Baillet-Guffroy A: Effect of UVA or UVB irradiation on cutaneous lipids in films or in solution. Photochem Photobiol 86: 553-562, 2010.

13. Kohl E, Steinbauer J, Landthaler M and Szeimies RM: Skin ageing. J Eur Acad Dermatol Venereol 25: 873-884, 2011.

14. Qin WZ, Zhu GD, Yang SM, Han KY and Wang J: Clinical observations on Tripterygium wilfordii in treatment of 26 cases of discoid lupus erythematosus. J Tradit Chin Med 3: 131-132, 1983.

15. Tao XL, Sun Y, Dong Y, Xiao YL, Hu DW, Shi YP, Zhu QL, Dai $\mathrm{H}$ and Zhang NZ: A prospective, controlled, double-blind, crossover study of Tripterygium wilfordii Hook F. in treatment of rheumatoid arthritis. Chin Med J (Engl) 102: 327-332, 1989.

16. Jiang X: Clinical observations on the use of the Chinese herb Tripterygium wilfordii Hook for the treatment of nephrotic syndrome. Pediatr Nephrol 8: 343-344, 1994.

17. Qiu D, Zhao G, Aoki Y, Shi L, Uyei A, Nazarian S, Ng JC and Kao PN: Immunosuppressant PG490 (triptolide) inhibits T-cell interleukin-2 expression at the level of purine-box/nuclear factor of activated T-cells and NF-kappaB transcriptional activation. J Biol Chem 274: 13443-13450, 1999.

18. Lin N, Sato T and Ito A: Triptolide, a novel diterpenoid triepoxide from Tripterygium wilfordii Hook. f., suppresses the production and gene expression of pro-matrix metalloproteinases 1 and 3 and augments those of tissue inhibitors of metalloproteinases 1 and 2 in human synovial fibroblasts. Arthritis Rheum 44: 2193-2200, 2001

19. Wu Y, Cui J, Bao X, Chan S, Young DO, Liu D and Shen P: Triptolide attenuates oxidative stress, NF- $\kappa \mathrm{B}$ activation and multiple cytokine gene expression in murine peritoneal macrophage. Int J Mol Med 17: 141-150, 2006.

20. Lu Y, Bao X, Sun T, Xu J, Zheng W and Shen P: Triptolide attenuates the oxidative stress induced by LPS/D-GalN in mice. J Cell Biochem 113: 1022-1033, 2012. 
21. Kabashima K, Nagamachi M, Honda T, Nishigori C, Miyachi Y, Tokura $\mathrm{Y}$ and Narumiya S: Prostaglandin E2 is required for ultraviolet B-induced skin inflammation via EP2 and EP4 receptors. Lab Invest 87: 49-55, 2007.

22. Sato T, Imai N, Akimoto N, Sakiguchi T, Kitamura K and Ito A: Epidermal growth factor and 1alpha,25-dihydroxyvitamin D3 suppress lipogenesis in hamster sebaceous gland cells in vitro. J Invest Dermatol 117: 965-970, 2001.

23. Akimoto N, Sato T, Iwata C, Koshizuka M, Shibata F, Nagai A, Sumida M and Ito A: Expression of perilipin A on the surface of lipid droplets increases along with the differentiation of hamster sebocytes in vivo and in vitro. J Invest Dermatol 124: 1127-1133, 2005.

24. Iwata C, Akimoto N, Sato T, Morokuma $\mathrm{Y}$ and Ito $\mathrm{A}$ : Augmentation of lipogenesis by 15-deoxy-Delta12,14-prostaglandin $\mathbf{J} 2$ in hamster sebaceous glands: Identification of cytochrome P-450-mediated 15-deoxy-Delta12,14-prostaglandin J2 production. J Invest Dermatol 125: 865-872, 2005.

25. Harris CA, Haas JT, Streeper RS, Stone SJ, Kumari M, Yang K, Han X, Brownell N, Gross RW, Zechner R and Farese RV Jr: DGAT enzymes are required for triacylglycerol synthesis and lipid droplets in adipocytes. J Lipid Res 52: 657-667, 2011.

26. Yamamoto T, Kurasawa M, Hattori T, Maeda T, Nakano $H$ and Sasaki H: Relationship between expression of tight junction-related molecules and perturbed epidermal barrier function in UVB-irradiated hairless mice. Arch Dermatol Res 300: 61-68, 2008.
27. Wróbel A, Seltmann H, Fimmel S, Müller-Decker K, Tsukada M, Bogdanoff B, Mandt N, Blume-Peytavi U, Orfanos CE and Zouboulis CC: Differentiation and apoptosis in human immortalized sebocytes. J Invest Dermatol 120: 175-181, 2003.

28. Kurihara H, Sato T, Akimoto N, Ogura T and Ito A: Identification and characterization of ABCB1-mediated and non-apoptotic sebum secretion in differentiated hamster sebocytes. Biochim Biophys Acta 1811: 1090-1096, 2011.

29. Trindade GS, Capella MA, Capella LS, Affonso-Mitidieri OR and Rumjanek VM: Differences in sensitivity to UVC, UVB and UVA radiation of a multidrug-resistant cell line overexpressing P-glycoprotein. Photochem Photobiol 69: 694-699, 1999.

30. Kang S, Cho S, Chung JH, Hammerberg C, Fisher GJ and Voorhees JJ: Inflammation and extracellular matrix degradation mediated by activated transcription factors nuclear factor-kappaB and activator protein-1 in inflammatory acne lesions in vivo. Am J Pathol 166: 1691-1699, 2005.

31. Sato T, Kurihara H, Akimoto N, Noguchi N, Sasatsu M and Ito A: Augmentation of gene expression and production of promatrix metalloproteinase 2 by Propionibacterium acnes-derived factors in hamster sebocytes and dermal fibroblasts: A possible mechanism for acne scarring. Biol Pharm Bull 34: 295-299, 2011.

32. Allen HB and LoPresti PJ: Acne vulgaris aggravated by sunlight. Cutis 26: 254-256, 1980. 